\title{
Modelo de Tecnologías de Información para la captación del talento deportivo en centros escolares peruanos
}

\section{Information Technology Model for attracting sports talent in Peruvian schools}

\author{
Arturo Laredo-Montero ${ }^{1, a}$, César Astete-Flor ${ }^{2, b}$, Jesús Mejía-Cavero ${ }^{3, c}$ \\ ${ }^{1}$ Universidad Nacional Mayor de San Marcos, Facultad de Ingeniería de Sistemas e Informática. Lima, Perú \\ $\underline{2}$ _Universidad Nacional Mayor de San Marcos, Facultad de Ciencias Económicas. Lima, Perú \\ ${ }^{3}$ Universidad Nacional Mayor de San Marcos, Facultad de Educación. Lima, Perú \\ a alaredom@unmsm.edu.pe \\ bcesar.astete1@unmsm.edu.pe \\ c11060288@unmsm.edu.pe
}

\begin{abstract}
Resumen
La presente investigación titulada "Modelo de Tecnologías de Información para la captación del talento deportivo en centros escolares peruanos" nace de la necesidad, por parte de instituciones estatales, de una herramienta de tecnología de información que ayude a las entidades competentes para mejorar la captación de competencias humanas en actividades deportivas y que los centros de educación escolar en el Perú carecen de este instrumento. Es por esta razón, que no todos los talentos son captados y los que se reclutan, en su mayoría, lo son por factores de suerte o voluntad propia. Esta investigación se realizó bajo el enfoque cualitativo y cuantitativo con un alcance descriptivo, donde se aplicó instrumentos como la entrevista y la observación a entrenadores, docentes de educación física, además de personal profesional involucrado en la gestión deportiva. Como producto de este estudio, se ha logrado perfilar un modelo de Tecnología de Información que permite integrar y desarrollar el talento deportivo a través de indicadores competitivos para medir sus destrezas y habilidades, que permitirán comprobar si el atleta captado tiene la capacidad deportiva para competir en los futuros torneos internacionales y reducir significativamente la deserción y pérdida de talentos.
\end{abstract}

Palabras clave: Información; tecnologías; talento; deporte; escuela.

\begin{abstract}
This research entitled "Information Technology Model for attracting sports talent in Peruvian schools" stems from the need, by state institutions, for an information technology tool that helps competent entities to improve in the capture of human skills in sports activities and that schools in Peru lack this instrument. It is for this reason, that not all talents are captured and those who are recruited, for the most part, are recruited by factors of luck or their own will. This research was carried out under the qualitative and quantitative approach with a descriptive scope, where instruments such as interviews and observation were applied to trainers, physical education teachers, as well as professional personnel involved in sports management. As a product of this study, it has been possible to outline an Information Technology model that allows the integration and development of sports talent through competitive indicators to measure their skills and abilities, which will allow to verify if the captured athlete has the sporting ability to compete in Future international tournaments and significantly reduced defection and loss of talent.
\end{abstract}

Keywords: Information; technologies; talent; sports; school.

Correspondencia:

Dirección: Dirección: Universidad Nacional Mayor de San Marcos, Facultad de Ingeniería de Sistemas e Informática. Calle Germán Amézaga N 375 , Ciudad Lima 1.

Recibido 03/09/2020 - Aceptado 10/12/2020 - Publicado: 21/12/2020

Citar como:

Laredo-Montero, A., Astete-Flor, C. \& Mejía-Cavero, J. (2020) Modelo de Tecnologías de Información para la captación del talento deportivo en centros escolares peruanos. Revista Peruana de Computación y Sistemas, 3(2):19-28. http://dx.doi.org/10.15381/rpcs.v3i2.19303

(C) Los autores. Este artículo es publicado por la Revista Peruana de Computación y Sistemas de la Facultad de Ingeniería de Sistemas e Informáticade la Universidad Nacional Mayor de San Marcos. Este es un artículo de acceso abierto, distribuido bajo los términos de la licencia Creative Commons Atribucion - No Comercia_Compartir Igual 4.0 Internacional. (http://creativecommons.org/licenses/by-nc-sa/4.0/) que permite el uso no comercial, distribución y reproducción en cualquier medio, siempre que la obra original sea debidamente citada. 


\section{Introducción}

En la actualidad, el deporte es tan importante para la vida humana como otras ciencias porque proporciona diferentes capacidades, además de las capacidades de aprendizaje (como el trabajo en equipo) concede las habilidades motoras, mejora la salud poblacional y se comporta como un ingrediente más contra el estrés en el estudiante. Es por eso, que también el estado debe poner énfasis en la orientación de los jóvenes hacia las disciplinas deportivas. A partir de ello, es conveniente realizar una gestión que tenga la responsabilidad de senalar aquellos indicadores que facilite la identificación del talento deportivo en los jóvenes estudiantes, con el fin de participar en competencias de alto nivel; para lograr este propósito con más exactitud se debe recurrir a las tecnologías de la información. Este conjunto de técnicas que se agrupan en un modelo de gestión asegura que el deportista sea elegido por sus capacidades y habilidades en una disciplina correcta.

Por otro lado, existe un escaso número de investigaciones que presentan nuevos modelos en base a tecnologías de información que permiten medir, de manera integral y correctamente, el proceso de datos que brinde indicadores eficientes para captar y seleccionar aquellos estudiantes que poseen el talento deportivo de una forma integral [1]. Esto debido a la poca resiliencia por parte del entrenador a usar las tecnologías, pues no saben cómo usarlas adecuadamente, registrando sus mediciones manualmente y, por parte de los deportistas que desconocen las ventajas si las aprovechan adecuadamente.

Por lo que también, es un desafío que enfrentan continuamente, las entidades dedicadas a la formación, desarrollo y competencia de las diferentes disciplinas deportivas. Para ello, se utilizan diversas estrategias de tecnologías de información y comunicación (TICs) donde se involucran muchas especialidades, no solo las de negocio, sino también en salud y deporte [2] En la actualidad se comprueba que el mal uso de las tecnologías de información en el deporte produce altas brechas en captar nuevos deportistas, debido a que generalmente las personas a cargo de las direcciones deportivas no están familiarizadas con la tecnología; por lo tanto, en la actualidad en su gran mayoría, registran información muy básica de solo datos antropométricos en un cuaderno, posteriormente transferidas manualmente a un hoja excel y posteriormente enviado por correo electrónico a las instancias competentes, conllevado en muchos casos a tener datos consolidados pero con error de información por digitación, empleando así mismo una verificación manual de los datos antropométricos por parte de los expertos, trayendo consigo decisiones erróneas o no exactas finalmente en la captación de deportistas calificados.

Si bien es cierto, cada vez más las TIC se integran al deporte, para que su integración sea efectiva, el asesoramiento adecuado del profesor de educación física debe ser más eficiente; sino, como dice Garzón [3], al no tener unos indicadores apropiados para captar y medir correctamente un talento deportivo, y no contar con tecnologías que ayuden a ese proceso de integrar indicadores eficientes, se pueden perder muchos deportistas calificados en el camino. También es una realidad que solo algunos llegan a consolidarse más por voluntad propia y factor suerte que por un sistema integral monitoreado, haciendo más rezagado el desarrollo del deporte peruano.

Actualmente el Instituto Peruano del Deporte [IPD] solo implementa sistemas de información para la parte administrativa y no para la gestión deportiva, ya que se desconocen sistemas estándares para los deportes. Además, las pocas consultoras de sistemas que han postulado para desarrollar una solución terminan desertando debido a que los propios involucrados desconocen el proceso de formación deportiva a un nivel necesario para modelar un sistema que, como señala Ramírez [4], las TICs que se utilizan en el deporte no tienen la integración madura entre la tecnología y los procesos de la organización deportiva.

En ese sentido, Moreno [5] determina que el abandono del deporte por parte de los deportistas en evaluación (o los postulantes que deseaban hacer carrera deportiva) se debe a que los modelos existentes para la captación del talento no eran satisfactorias, ante lo cual recomendó realizar nuevos modelos apoyados por la tecnología con el fin de evitar perder talentos, tal es el caso de un deportista seleccionado que terminó abandonando el deporte dedicándose a la actividad de ludopatía, un problema psicológico que no fue determinado al inicio, y posiblemente se descartó en su lugar a otros talentos con menos problemas socio-culturales en el Centro Experimental Deportivo (CEDE) del Instituto Peruano del Deporte (IPD).

Ante todos estos problemas, se suma una más, que no será de estudio profundo en esta investigación pero que vale recalcar, para futuros trabajos, y es que "las instalaciones deportivas pueden ser uno de los escollos más importantes a la hora de planificar un campus. En este sentido, se debe analizar qué tipo de campus se puede abarcar, con qué capacidad y con qué horarios. [6]

Esto nos lleva a reflexionar que los modelos actuales de captación de talentos en el Perú, por parte de los entrenadores o especialistas realizan esta actividad de forma visual, sin apoyo de ninguna métrica estándar ni sistema de información que filtre algún dato erróneo, tal como lo indica el propio director del Centro Experimental Deportivo, indicando que "si existiera indicadores respecto a la evolución en línea de uno de mis chicos a lo mejor a ninguno los perdería".

Por lo tanto, existe deficiencias en la captura de información porque es manual, como se describe en la figura 1, por lo que esta información no es validada ni filtrada, ni está disponible. Esto termina por tomar decisiones erróneas y poco acertadas respecto a la gestión deportiva en una institución que forma talentos deportivos. 


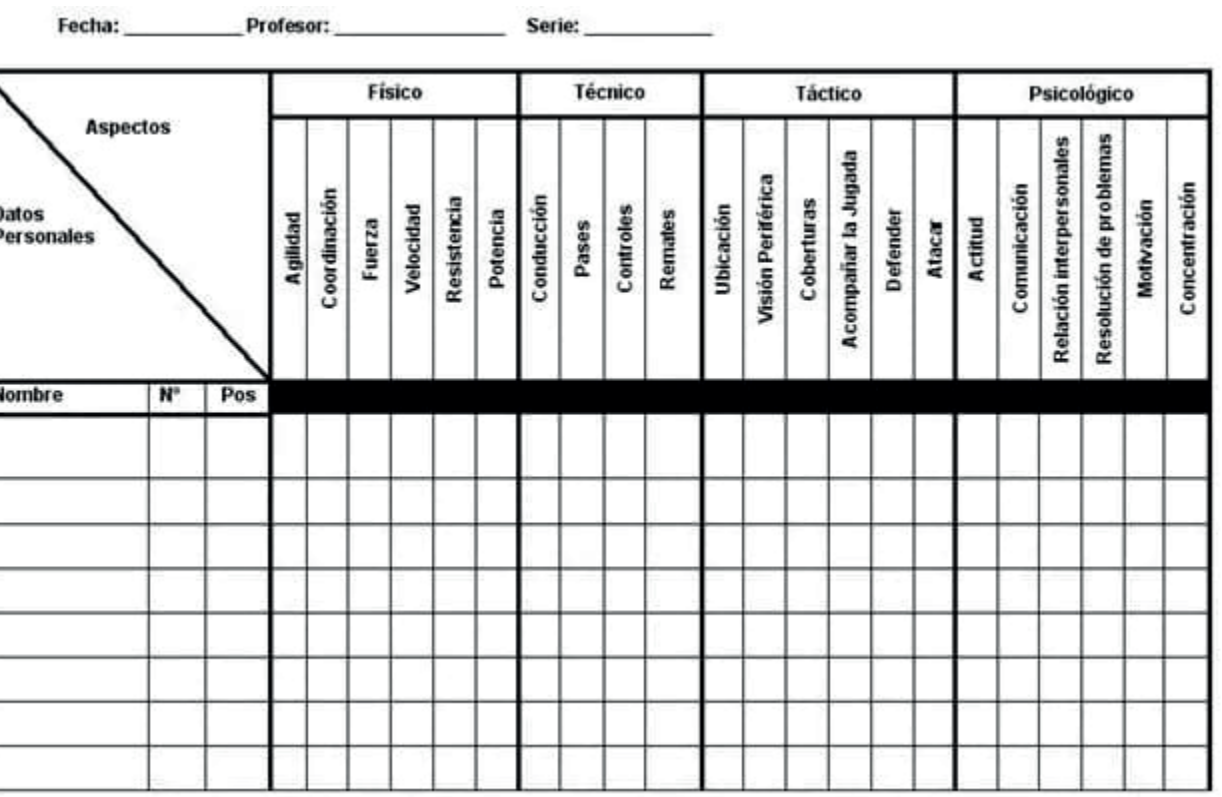

Fig. 1. Ficha manual para evaluación deportiva.

Fuente. Dirección Nacional de Recreación y Promoción del Deporte - IPD.

Existe poca información sobre un modelo de tecnologías que midan integral y correctamente los procesos y datos de la gestión deportiva de manera eficiente y oportuna que permitan captar, desarrollar y producir más talentos deportivos.

Así mismo, los entrenadores no se comportan como formadores de talento, ni utilizan indicadores multidisciplinarios que puedan elaborar un diagnóstico integral con proyección de desarrollo, que no solo se limiten en medidas antropométricas, sino que estén establecidas en la evaluación deportiva integrada con tecnología de información. [7]

La presente propuesta de modelo que contenga Tecnologías de la Información (TI) para la gestión deportiva, servirá como base para estandarizar de manera óptima la medición e integración de los indicadores deportivos que en la actualidad se realizan por separado y con escaso tratamiento de datos, por lo que también servirá como punto de partida la creación de un modelo único de TI que garantice efectivamente la consolidación de gestionar el rendimiento para lograr el éxito competitivo en todos los deportes.

Este trabajo está seccionado en cinco partes: En la primera, elabora una introducción donde se resume la situación actual y la importancia del modelo; en la segunda, se realiza la revisión de la literatura sobre las distintas tecnologías de información y su aporte a la ciencia del deporte. En la tercera sección se realiza la metodología seguida para detallar la construcción del modelo propuesto, así su validez para desarrollarlo. En la cuarta sección se presenta el modelo propuesto con el resultado empírico verificando la estructura y funcionalidad del modelo planteado. Finalmente, en la quinta sección se presentan las conclusiones de la investigación.

\section{Revisión de la literatura}

Existen estudios sobre la relación de las TICs con el Deporte y de qué manera éstas influyen en el rendimiento o no. Es así como estudios que realizaron Tejero, Castro y Balsalobre [1] en la Universidad de Santiago de Compostela de España resume que "tradicionalmente la relación que existía entre las Tecnologías de la Información y de la Comunicación y el deporte era muy escasa inicialmente". La mayor parte de su aplicación en el mundo del deporte se reducía al ámbito del rendimiento deportivo. Es decir, solo a la alta competitividad, pero no a los procesos iniciales de formación deportiva como la captación de talentos.

Si sumamos a que en la actualidad no existe suficiente documentación sobre modelos de gestión deportiva podemos entender que cada país o comunidad diseña a su manera la mejor estructura posible para poder gestionar su deporte, y a su vez éstas adaptan la tecnología como mejor sea posible también. [8].

Afortunadamente, en la actualidad la situación está cambiando y las TIC ofrecen ahora una gran variedad de avances que son empleados y adaptados en otras materias para complementar las actividades y conocimientos que se les imparten a los deportistas para medir su rendimiento; y debido a la poca información de modelos de TI aplicados a la gestión deportiva, se ha encontrado estudios de innovaciones tecnológicas destinados para funciones distintas en su origen y que son aplicados a la medicina, por ejemplo, APPS para medir el stress laboral. [9]

Tal es el caso, del estudio de Cerón, et al sobre "Estrategias basadas en tecnologías de la información y la comunicación para la reducción de factores de riesgo 
cardiovascular en personas laboralmente activas", donde afirma que existe experimentación en el uso de aplicaciones móviles para problemas de salud cuando han sido creadas para otro propósito. [10].

Pero a medida que se implantan metodologías, modelos y se desarrollan aplicaciones tecnológicas para poder medir correctamente el rendimiento y optimizar el performance del deportista, aún no se logra que las TIC se integren de tal manera que realmente contribuyan a impulsar el rendimiento de manera óptima. Un estudio sobre "El desarrollo de las competencias TIC en los contextos formativos" determina que los estudiantes no llegan a ser competentes en cuanto al uso de las TICs, por lo tanto, los atletas en formación tampoco utilizan correctamente la tecnología en su propio beneficio [11]. Uno de los motivos es el ingreso de información que no está orientada y clasificada correctamente para la formulación de indicadores, lo cual imposibilita a las TICs mostrar los resultados que sean idóneos en la construcción de un modelo que permita ver las cualidades más resaltantes del deportista.

Se debe tener en cuenta que no todo es simple en la implementación de las TIC's en el desarrollo del deporte, ya que se necesitan de la fusión de todos los especialistas de distintas especialidades y no solo deportivas para poder realizar un muestreo de datos que realmente sirvan para una buena medición y tomar decisiones acertadas tanto para la evaluación del talento escondido o del nato que recién denota alguna excepción, sino también de los deportistas que en el camino necesitan saber de manera adecuada su propia evolución. Como lo recomienda García S., Xabel en su estudio sobre "el Papel del Perfil Resiliente y las Estrategias de Afrontamiento sobre el estrés-recuperación de los deportistas de competición”, que aboga por la inclusión de otros tipos de controles como los de psicología y que han demostrado mucha eficacia en los resultados finales. [12].

Este proceso no depende del todo de las TICs si es que no se diseñan estructuras de datos de tal manera que indiquen el real estado y síntomas del rendimiento. Anticiparse y prever la evolución o involución de un deportista es el reto que muchos autores dedican actualmente al estudio del cual dependen muchos deportistas que han sido ignorados por una mala captación y monitoreo, va más allá de una visión estática del proceso de tecnología deportiva adoptando un enfoque más dinámico y flexible. Por eso, las organizaciones deportivas han tenido que cambiar significativamente la forma en que prestan sus servicios. [13]

Este problema también contribuye a que las TICs no se integren en un solo modelo de captación de talento deportivo porque los profesores de educación física, en su mayoría, prefieren seguir usando sus métodos tradicionales de medición aun sabiendo que son más próximos al error, pero se sienten más familiarizados. [14].
Sin embargo, Molina et al, mencionan que las demandas de tecnologías siguen llegando al deporte por lo que "las TIC son una de las herramientas más atractivas para conseguir datos relevantes" [15] y los docentes de educación física, cada vez más, utilizan tecnologías de la información y comunicaciones en sus clases, pero su uso aun no es significativo ya que lo utilizan mayormente para gestiones administrativas y no de medición, además, el texto de Alvarado [16] resume que las TIC pueden aportar mucho más al desarrollo del deporte, pero todo dependerá de su correcto uso tanto por los propios deportistas, profesores de educación física y todos los involucrados en la gestión deportiva.

\subsection{Tecnologías de la Información para el Deporte.}

Actualmente, los adelantos tecnológicos lo notamos con más frecuencia usualmente en cualquier tema y, desde luego, el deporte no podía ser una distinción.

Estas herramientas mejorarían el rendimiento deportivo del atleta para conseguir mejores exámenes y así lograr ser más competitivos en la disciplina que practica, marcando la diferencia en aquellos pequeños detalles tan difíciles de alcanzar. [17].

Estas nuevas tecnologías cambiarán conforme se implementen para cada disciplina deportiva. Sin embargo, no son aplicadas de forma adecuada para la formación de un deportista y no demandan muchas innovaciones para la captación, la tendencia tecnológica va más por la élite y el alto rendimiento. [18].

\subsection{Tecnologías de Información aplicables en Educación Física.}

2.2.1. Tecnología Inmersiva: Esta tecnología con sistemas ciber-físicos avanza rápidamente. Las experiencias de realidad virtual (VR) y realidad aumentada (AR) llegan a comercializarse rápidamente al público en general, incluidos los aficionados al deporte. Varios intentos se han diseñado para utilizar la tecnología de realidad virtual con el fin de experimentar y disfrutar deportes que podrían ser costosos, peligrosos o de difícil acceso debido a limitaciones geográficas. Por ejemplo, los simuladores de equitación de realidad virtual se desarrollado con fines educativos y ahora cualquiera puede disfrutar de forma segura de deportes extremos como ciclismo de montańa y paracaidismo a través de la realidad virtual [19]. A este modelo también se le denomina realidad mixta, pues el término se ha usado para la simulación de realidad, creando el efecto inmerso.

\subsubsection{Tecnología de Captura de Movimiento:}

La web del instituto especializado en diseño digital Arteneo menciona que "la Captura de Movimiento, MOCAP, [del inglés motion capture, o motion abreviada mocap] es una técnica de grabación de movimiento de actores y animales vivos trasladando dicho movimiento a un modelo digital, realizado en imágenes de computadora”. [20]. 
2.2.3. Big Data: Ramírez [4] menciona que "Big Data es un término que describe el gran volumen de datos, tanto estructurados como no estructurados, que inundan los negocios cada día”. Pero lo que interesa con este modelo como se organizan los datos. Esta tecnología permite a los profesores organizar una vasta cantidad de datos de muchas evaluaciones, inclusive al mismo tiempo y enviarlas a una nube o a una base de datos de almacenamiento que se encontraría en el IPD.

2.2.4. Modelo Cloud IT.: Moreno [5] define a Cloud computing [Computadora en la nube] como un "nuevo modelo de prestación de servicios de negocio y tecnología, que permite incluso al usuario acceder a un catálogo de servicios estandarizados y responder con ellos a las necesidades, de manera flexible y adaptativa”. La idea de esta tecnología es que los profesores transfieren los datos desde una nube digital desde cualquier punto del país a la base.

2.2.5. Telepsicología: La Telepsicología según Brown citado en Botella, et al [6] consiste en:

"el uso la tecnología de las telecomunicaciones para poner en contacto a los pacientes con los profesionales de la salud con el propósito de posibilitar un diagnóstico efectivo del paciente"; asimismo, puede ser una contribución para la investigación y otras actividades vinculadas con los intereses del sector salud. [11, p25]. No todas las personas tienen la misma capacidad de interactuar y/o enfrentar situaciones adversas, por lo que los deportistas deben ser evaluados constantemente, pero a traves de la tecnologia, permitirá hacer escalas psicológicas mas precisas y en tiempo record, lo que ayudará al profesional de salud trabajar el o los aspectos que crea conveniente desde cualquier región y enviar informes de forma simultánea.

\section{Metodología propuesta}

Para el modelo propuesto se identificó una serie de indicadores que se obtienen de la ficha que brinda el IPD para la captación de talento en los deportes colectivos e individuales. Una vez que se tiene estos indicadores, se pasa a describir las fases de la metodología que se utiliza para el presente modelo descrito en tres fases, lo cuales se ven en la figura 2:

De acuerdo con la metodología para diseñar este modelo, se dividieron en tres fases, que son:

Fase 1. Análisis: Se selecciona los indicadores, como parte de recolección de información, que serán extraídos de la ficha de evaluación que brinda el IPD, mediante su manual técnico metodológico que se dispone de los "Lineamientos para la Organización y Desarrollo de las Actividades". [21]. Finalmente, se desprende qué indicadores antropométricos (físicos) tácticos y psicológicos se usan para ver en qué disciplina(s) se desarrollaría el nuevo deportista.

Fase 2. Evaluación: En esta fase, se coloca en una matriz de doble entrada (Fig. 3) las tecnologías de información que sirven para el modelo que pueden ayudar a captar talento en los aspectos físicos, técnicos, tácticos y psicológicos del joven deportista.

Fase 3. Diseño: se transparenta la información de manera eficiente siguiendo los 4 aspectos de evaluación de talento y se halla la tecnología más idónea para evaluarlo. Así se elabora el diseño del modelo de TI para la capacitación del evaluador de Educación Física y su aplicación en la captación de talento deportivo.

Por otro lado, la metodología de investigación se cińe a un tipo Aplicado, pues aplica aspectos teóricos para obtener un resultado. Esta investigación permite

Fase 1: Análisis

Fase 2: Evaluación

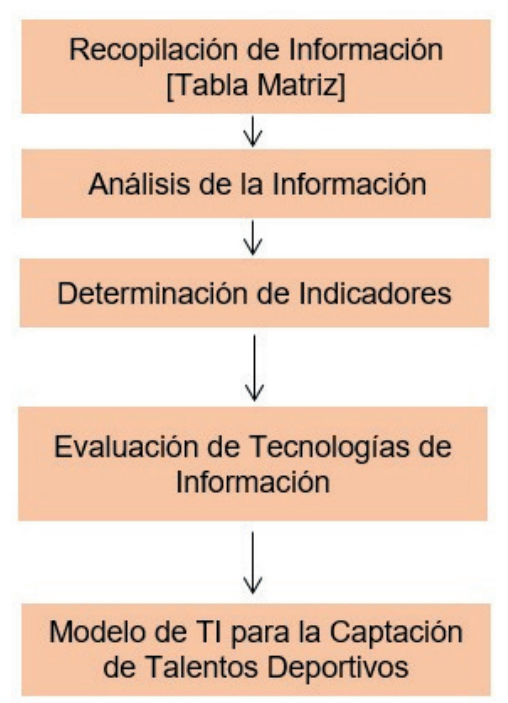

Fig. 2. Metodología para la definición del Modelo de tecnologías de información en captación de talentos deportivo. 


\begin{tabular}{|c|c|c|c|c|c|c|c|}
\hline \multicolumn{2}{|c|}{$\begin{array}{l}\text { Aspectos de } \\
\text { evaluación - } \\
\text { Talento Deportivo }\end{array}$} & 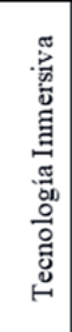 & 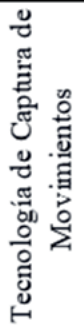 & 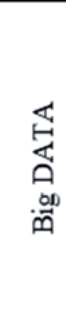 & 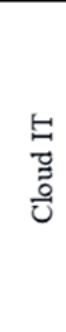 & 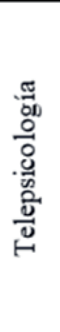 & 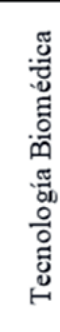 \\
\hline \multirow{6}{*}{ Fisico } & Agilidad & $\times$ & $\times$ & $x$ & $\times$ & & \\
\hline & Coordinación & $x$ & $x$ & $x$ & $x$ & & $\mathrm{x}$ \\
\hline & Fuerza & $\mathrm{x}$ & $x$ & $x$ & $x$ & & $\mathrm{x}$ \\
\hline & Velocidad & $x$ & $\mathrm{x}$ & $x$ & $x$ & & $\mathrm{x}$ \\
\hline & Resistencia & $\mathrm{x}$ & $x$ & $x$ & $x$ & & $\mathrm{x}$ \\
\hline & Potencia & $x$ & $x$ & $x$ & $x$ & & $x$ \\
\hline \multirow{4}{*}{ Técnico } & Conducción & $\mathrm{x}$ & $x$ & $x$ & $x$ & & $\mathrm{x}$ \\
\hline & Pases & $x$ & $x$ & $x$ & $x$ & & $x$ \\
\hline & Controles & $x$ & $x$ & $x$ & $x$ & & $\mathrm{x}$ \\
\hline & Remates/tiros & $\mathrm{x}$ & $\mathrm{x}$ & $x$ & $x$ & & $\mathrm{x}$ \\
\hline \multirow{6}{*}{ Táctico } & Ubicación & $x$ & $x$ & $x$ & $x$ & & $\mathrm{x}$ \\
\hline & $\begin{array}{c}\text { Visión } \\
\text { Periférica }\end{array}$ & $\mathrm{x}$ & $\mathrm{x}$ & $\mathrm{x}$ & $\mathrm{x}$ & & $\mathrm{x}$ \\
\hline & Cobertura & $x$ & $x$ & $\mathrm{x}$ & $\mathrm{x}$ & & $\mathrm{x}$ \\
\hline & $\begin{array}{c}\text { Acompañar la } \\
\text { jugada }\end{array}$ & $\mathrm{x}$ & $\mathrm{x}$ & $\mathrm{x}$ & $\mathrm{x}$ & & $\mathrm{x}$ \\
\hline & Defender & $x$ & $x$ & $x$ & $x$ & & $x$ \\
\hline & Atacar & $x$ & $\mathrm{x}$ & $\mathrm{x}$ & $\mathrm{x}$ & & $\mathrm{x}$ \\
\hline \multirow{6}{*}{ Psicológico } & Actitud & & & & $x$ & $x$ & \\
\hline & Comunicación & & & & $x$ & $x$ & \\
\hline & $\begin{array}{c}\text { Relación } \\
\text { interpersonal }\end{array}$ & $\mathrm{x}$ & & & $\mathrm{x}$ & $x$ & \\
\hline & $\begin{array}{c}\text { Resolución de } \\
\text { problemas }\end{array}$ & $x$ & & & $\mathrm{x}$ & $\mathrm{x}$ & \\
\hline & Motivación & $x$ & & & $x$ & $x$ & \\
\hline & Concentración & $\mathrm{x}$ & & & $\mathrm{x}$ & $\mathrm{x}$ & \\
\hline
\end{tabular}

Fig. 3. Matriz de relación entre las TI e indicadores (Aspectos) de evaluación para captar talento deportivo.

crear las condiciones necesarias o adecuar las existentes a través del esclarecimiento de los recursos óptimos de la Tecnología de Información, que son de utilidad para integrar a los indicadores para captar talento, pues la finalidad de esta publicación es la de diseñar un Modelo de TI con gestión de Indicadores que permita descubrir cuáles son las habilidades atléticas que todo chico debe tener para cierta disciplina deportiva, sea colectiva y/o individual. Asimismo, la presente investigación tiene un Enfoque cualitativo ya que se integrará en el instrumento los tipos de indicadores de manera cualitativa.

\subsection{Aplicación de las Tecnologías de Información.}

Dentro de Tecnologías aplicadas a la captación de talento deportivo se ha revisado seis, que son los más importantes:
3.1.1. Tecnología Inmersiva: Como tecnología es muy usado en simulaciones de información por parte de entrenadores y captadores de talento en sistemas de información que proporcionan datos, estadísticas y rankings, que permite la realidad mixta para uso deportivo. Estas tecnologías predominan en el ambiente de alto rendimiento porque, en algunos casos, se obtiene las últimas novedades tecnológicas respecto a las competiciones internacionales y sobre todo por otorgar información de cómo debería ser los entrenamientos y dedicación del deportista, que en muchos casos, el efecto del sentido de presencia sobre la excitación emocional y la actitud utilizando neurofisiología y medidas psicométricas y evaluar el comportamiento futuro del deportista. (22).

3.1.2. Tecnología de captura de movimientos: Este tipo de tecnologías se empezaron a usar en la industria del cine, ya que recrea y traslada dicho movimiento 
físico y real a un espectro digital que puede ser después estudiado y caracterizado para transformarlo en otra entidad. Su tecnología se basa en la fotogrametría, por lo que también es usada en la telemedicina y en el deporte de alto rendimiento.

3.1.3. Big Data: Es una de las tecnologías que está revolucionando para el análisis de datos. En el mundo deportivo no se usa mucho ya que se reconoce más en el ambiente de transferir grandes cantidades de datos. Los grandes clientes de estas tecnologías son empresas que buscan captar datos más actuales y con rapidez.

3.1.4. Cloud IT: El almacenamiento virtual es una de las formas más eficaces en la actualidad que demanda el resguardo de información de manera inmediata y en línea. Muy usado en todo tipo de profesiones, incluyendo en el deporte.

3.1.5. Telepsicología. "La Telepsicología presta servicios a distancia a través de múltiples tecnologías, como pueden ser Internet, a través de chat y videoconferencias, y software, instalados en el ordenador y en el móvil". Todo ello basado en un intercambio de información detallado entre el psicólogo y el cliente [en este caso un deportista] y el desarrollo de unas sesiones estructuradas. Debido a la diferencia entre este contexto y el llevado a cabo en una consulta física, son necesarios diferentes procesos terapéuticos.

Algunos ejemplos de programas empleados son la ciberterapia a través de la realidad virtual $[\mathrm{RV}]$, la tele rehabilitación o programas auto aplicados y test basados en tecnología web.

Es importante diferenciar entre la Telepsicología y la "psicología online". Esta última consiste en sitios web mecanizados e independientes de un psicólogo que proporcionan información sobre psicología, contienen test, foros de discusión, etc. Algunos de ellos prestan ayuda psicológica, pero de forma leve. La principal diferencia es que estas webs son gratuitas, a diferencia de la Telepsicología, que pretende ser lo más parecida posible a la psicología que ofrecen los profesionales en sus consultas.

Actualmente existen programas de Telepsicología que se han aplicado con éxito a distintos problemas de falta de acompañamiento, seguimiento, prevención, diagnósticos en salud, e incluso, el desarrollo de este campo es tal, que empiezan a aparecer trabajos en los que se analizan las ventajas que producen este tipo de intervenciones y cómo influyen directamente en la salud y bienestar en los escolares y deportistas en plena formación. La Telepsicología va en aumento, un estudio publicado por la "American Psychological Association" en marzo del 2010 demostró que esta rama de la psicología ha ido expandiéndose en la última década, y se espera que en los próximos años este progreso continúe y se expanda a nivel mundial para diversas disciplinas, en este caso, porque no en la gestión deportiva para el proceso de captación y formación.
3.1.6. Tecnología Biomédica: La Tecnología Biomédica "es uno de los campos de actividad de mayor crecimiento de los últimos ańos y su desarrollo ha permitido mejorar sustancialmente la salud de las personas" a partir de técnicas de diagnóstico y tratamiento que eran inimaginables hace pocos años atrás de una manera rápida y en línea.

Las áreas de aplicación de la tecnología biomédica van desde el sector salud hasta del deporte a través de la medicina deportiva, que acelera el proceso de recuperación de atletas tras una lesión por citar un ejemplo, así como el monitoreo y seguimiento para evaluar el rendimiento del deportista y grado de evolución en cuanto a su condición física.

\section{Modelo Propuesto.}

Para el modelo propuesto, se seleccionó los indicadores para la captación del talento deportivo que se determinó en la Fase 1, durante las actividades de "Recopilación y Análisis de Información” [Fig. 2]. Dichas tecnologías se seleccionaron para demostrar la utilización en el ambiente deportivo, por lo que sirvieron como base para la propuesta de modelo de TI con los elementos de evaluación para la gestión deportiva, como se muestra en la siguiente Tabla 1.

Al evaluar las distintas tecnologías del modelo para validar su instrumento se obtuvo una serie de puntajes dicotómicos $(0,1)$ que se le brinda a cada tecnología para los elementos que lo analizarán, donde $0=$ No y 1 = Sí, con el propósito de obtener una validez por medio de la prueba $V$ de Aiken (Ecuación 1) que optimice el buen desarrollo del modelo con sus indicadores.

Le estimación de esta ecuación permite calcular un nivel de probabilidad válido para la adecuación del contenido del instrumento que se va a usar, en este caso las tecnologías de información bajo los aspectos de evaluación que se encuentran en la primera columna de la tabla 1.

$$
V=\frac{X-l}{k}
$$

$X=$ media de calificaciones de los jueces

I = valor de calificación más bajo de la escala

$\mathrm{k}=$ rango de los valores posibles de la escala

Ecuación 1. V de Aiken

Así, se tiene una validez en V de Aiken de 0.722, lo cual indica que las tecnologías propuestas tienen una alta validez de aplicación en la captación del talento deportivo.

\subsection{Diseño del modelo TI en captación de Talento Deportivo.}

El siguiente modelo de TI reúne las tecnologías de información seleccionadas que convergen con los procesos de la captación del talento deportivo, que se encuentra en la siguiente figura 4: 
Tabla 1. Elementos de Evaluación de la Tecnología de Información en la Captación de Talento Deportivo

\begin{tabular}{|c|c|c|c|c|c|c|}
\hline Evaluación de TI en Captación de Talentos & 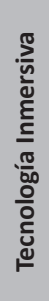 & 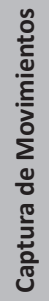 & $\begin{array}{l}\frac{\varangle}{6} \\
\frac{000}{0} \\
\frac{00}{0}\end{array}$ & $\begin{array}{l}\text { ㄴ } \\
\text { ס्र } \\
\frac{0}{\cup}\end{array}$ & $\begin{array}{l}\frac{\pi}{00} \\
\frac{0}{8} \\
\frac{u}{n} \\
\frac{0}{d} \\
\stackrel{0}{L}\end{array}$ & 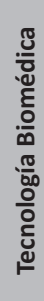 \\
\hline Distribución de Contenidos & 1 & 1 & 1 & 1 & 0 & 1 \\
\hline Manejo de Estrategias & 1 & 1 & 1 & 0 & 1 & 1 \\
\hline Seguimiento al deportista & 1 & 1 & 1 & 0 & 1 & 1 \\
\hline Medir salud mental & 0 & 0 & 0 & 0 & 1 & 1 \\
\hline Medir rendimiento físico & 1 & 1 & 0 & 0 & 1 & 1 \\
\hline Disponibilidad de información para el entrenador y deportista & 1 & 1 & 1 & 1 & 0 & 1 \\
\hline
\end{tabular}

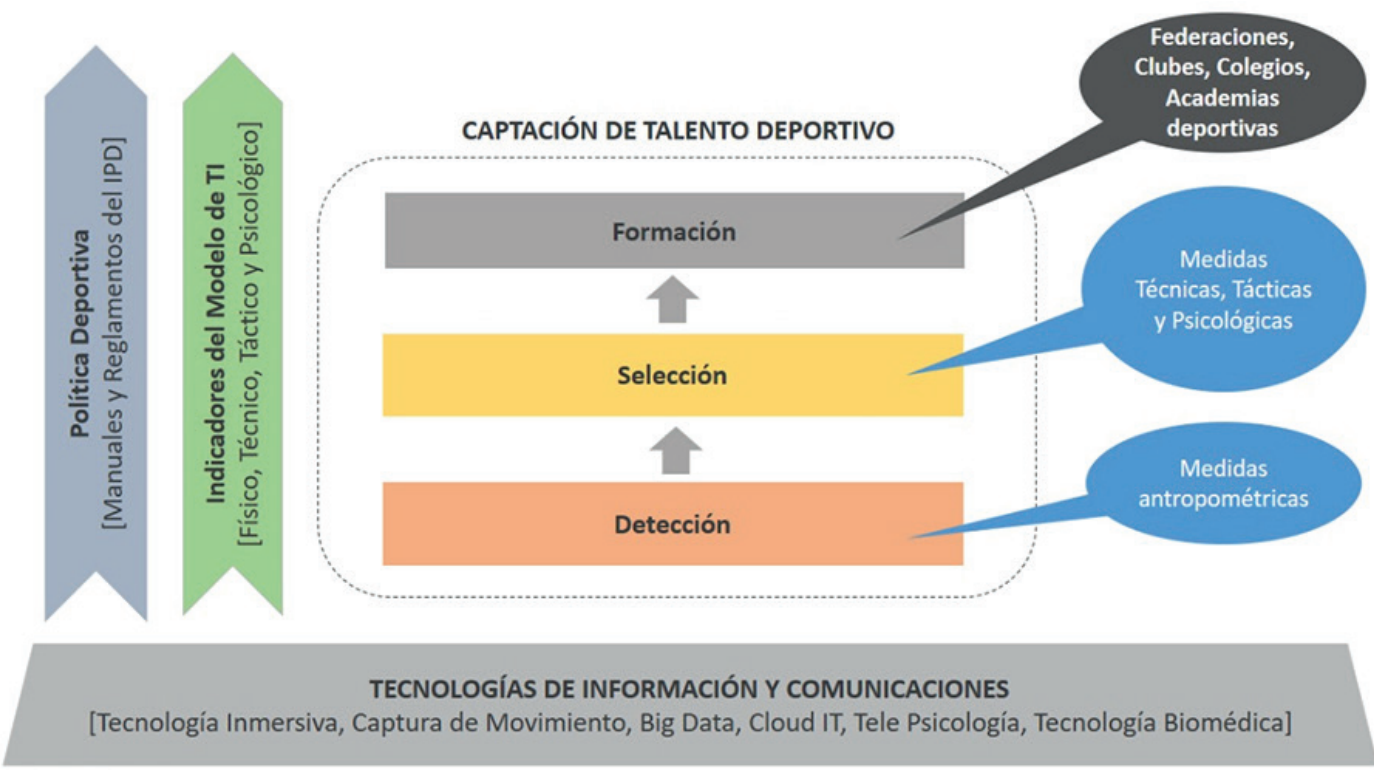

Fig. 4. Diseño del Modelo de TI propuesto para la Captación de Talento deportivo.

Después de haber completado el diseño del Modelo de TI que se basa en los indicadores que usa la Escuela Experimental de Deportes "CEDE" del IPD - Instituto Peruano del Deporte" del Perú, partiendo de la figura 4, se muestra el dinamismo de las tecnologías que brinda este modelo para la detección de talento deportivo.

De la Figura anterior, se puede apreciar que la primera fase del modelo es la "Detección" (de talento deportivo), donde los postulantes son evaluados mediante una serie de indicadores de medición antropométrica para evaluar su condición física. Para este fin se usa tecnologías como Big data y Cloud IT, donde se almacena y luego se transfiere información en tiempo real, a través de smartphones con aplicaciones instaladas que envían datos con mejor precisión (y en segundos) hacia el servidor principal que estará en las instalaciones del IPD, minimizando considerablemente el tiempo que los entrenadores usaban anteriormente para medir y enviar estos datos de cada postulante.
Para la segunda fase "Selección", se tuvo que emplear más Tecnologías, puesto que se distingue los distintos deportistas que van a practicar las múltiples actividades deportivas que se tienen en cada una de sus federaciones. En esta etapa, se evalúa los indicadores técnicos, tácticos y psicológicos del deportista, ya sea para disciplina individual y/o colectiva. En esta etapa se dispone de las distintas tecnologías que se mencionaron anteriormente.

- Tecnología inmersiva y captura de movimientos, se usará para medir las distintas medidas técnicas y tácticas, a fin de recrear los movimientos exactos (como velocidad, fuerza, proyección, defensa y ataque) del postulante. Para esto, se generará resiliencia en los entrenadores al ser capacitados previamente.

- Big data y Cloud IT, donde los datos generados por los anteriores indicadores quedarán almacenados en una base de datos y seguirá el mismo proceso de la primera fase. 
- Telepsicología y Biomedicina, por lo que no todos están aptos para las exigencias que requiere cada disciplina. Además de estar físicamente bien, lo debe de estar mentalmente. Pues se ha visto casos de deportistas que son muy buenos físicamente, pero caen en vicios y no saben afrontar situaciones adversas y/o estresantes.

Para la tercera fase "Formación", se incluye a los especialistas deportivos que se encuentran dentro de las federaciones, se encargarán de comenzar el periodo de "Iniciación deportiva" donde se da los primeros entrenamientos, y donde los formadores comienzan a interiorizarse en la realidad de cada postulante en los aspectos social, cultural y económico y cómo pueden superar obstáculos extradeportivos.

\section{Resultados y conclusiones.}

Se evaluaron las tecnologías de información que permiten la captación del talento deportivo con los indicadores adecuados y se identificaron diferentes indicadores para adaptar a cada talento.

El modelo de TI para la captación de talento deportivo fue diseñado sobre la base de tecnologías ya dedicadas al mundo de los negocios como Tecnología inmersiva, Big DATA y Cloud TI, así como las dedicadas al campo de las organizaciones no deportivas, pero que establecen sinergias entre sus propias funciones y que a su vez también son compatibles con las tecnologías en ciencias de la salud como la psicología [Telepsicología] y la medicina [Tecnología Biomédica] que han servido para integrarse en el arquetipo propuesto.

Los nuevos indicadores para captar el talento deportivo se establecieron mediante la integración de estas tecnologías en un nuevo modelo de TI, donde los nuevos criterios de evaluación usados de otras disciplinas y que convergen con la salud y la psicología de un deportista, estos también apoyan un mejor análisis y la toma de decisiones a través de la integración de información de multi disciplinas.

Con la aplicación de las Tecnologías, también se benefician los profesores de educación física de CEDE-IPD, ya que tendrán más facilidad para la evaluación de los procesos de captación y selección de talentos deportivos y serán mejor abordados en sus procesos de análisis de talentos. Muchos postulantes de diferentes provincias y distritos del interior del país pueden ser seleccionados como talentos deportivos con el nuevo criterio de evaluación apoyados con el modelo de TI propuesto. Por lo tanto, el número de atletas convocados aumentarán y serán derivados a selecciones y federaciones, teniendo más deportistas calificados y de alto rendimiento.

\section{Referencias Bibliográficas.}

[1] Balsalobre C., Tejero CM, Castro M. The importance of effect size: a statistical example using physical condition measurement. Revista Internacional de Medicina y Ciencias de la Actividad Física y el Deporte. 2015; 12(48): p. 715-727.
[2] Sotiriadou P. Mejorando la experiencia práctica en la gestión deportiva: un estudio de caso. European Sport Management Quarterly. 2011; 11(5): p. 525-546.

[3] Garzón C. Coevolución de las TICs con la Estrategia del Negocio. 2015; 23(33).

[4] Ramírez N. L. Campeones de la Transformación Digital: 10 líderes españoles Madrid: Profit Editorial; 2018.

[5] Moreno M. Computación en la nube. CEMA. 2015 junio;(566): p. 1-17.

[6] Botella C, Baños R, Garcia-Palacios A, Quero S, Guillén V, Marco H. La utilización de las nuevas tecnologías de la información y la comunicación en psicología clínica. UOC Papers. 2007;(04): p. 32-41.

[7] Muñoz FL, Díaz GE, Gallego RS. Las responsabilidades derivadas del uso de las tecnologías de la información y comunicación en el ejercicio de las profesiones sanitarias. Anales de Pediatría. 2020; 92(5): p. 301-307.

[8] Moreno P. Y. Modelo de gestión deportiva para el municipio de Quibdó. ELSEVIER. 2014 Diciembre; 5(12): p. 75-194.

[9] Carballo M, Barrero M, Villazón A. FiMAN: Sistema Computarizado para Analisis de Movimientos Digitales. Investigación y Desarrollo. 2016.

[10] Cerón J, López D, Urbano L, Alvarez-Rosero R, Zulma L. Estrategias basadas en tecnologías de la información y la comunicación para la reducción de factores de riesgo cardiovascular en personas laboralmente activas. Revista Colombiana de Cardiología. 2018 Enero; 25(01): p. 92-100.

[11] Humanante-Ramos P, et al. Las competencias TIC de los estudiantes que ingresan en la universidad: una experiencia en la Facultad de Ciencias de la Salud de una universidad latinoamericana. Educación Médica. 2018 Febrero;: p. 319-380.

[12] García X, Salguero A, Molinero O, De la Vega R, Ruiz R, Márquez $\mathrm{S}$. El papel del perfil resiliente y las estrategias de afrontamiento sobre el estrés-recuperación del deportista de competición. Kronos. 2015; 14(1).

[13] Ratten V. Sport technology: A commentary. The Journal of High Technology Management Research. 2020; 31(1).

[14] Fernandez E. C, Ladrón de Guevara L. El uso de las TIC en la Educación Física actual. E-motion. Revista de Educación, Motricidad e Investigación. 2015 Enero;(5): p. 17-30.

[15] Tiching. Las TIC en la Educación Física. [Online].; 2013. Available from: http://blog.tiching.com/las-tic-en-la-educacion-fisica/.

[16] Alvarado P. C. Uso de las TICs por parte de los profesores de Educación Física. EFDeportes.com, Revista Digital. 2017.

[17] Briceño A. Deber de Tecnologias. Revista Digital SCRIBD. 2015;: p. $1-4$.

[18] Roberts AH, Greenwood DA, Stanley M, Humberstone C, Iredale F, Raynor A. Coach knowledge in talent identification. A systematic review and meta-synthesis. Journal of Science and Medicine in Sport. 2019; 22(10): p. 1163-1172.

[19] Plante TG, Bodgen R, Bogden R, Canelin C. Might virtual reality promote the mood benefits of exercise? Computers in Human Behavior. 2003; 19(4).

[20] Arteneo. Captura de movimiento o motion capture: ¿cómo funciona? [Online].; 2015 [cited 2019 Enero 25. Available 
from: https://www.arteneo.com/blog/captura-de-movimientos-motion-capture-como-funciona/.

[21] Instituto Peruano del Deporte (IPD). Lineamientos para la Organización y Desarrollo de las Actividades Lima: Dirección Nacional de Recreación y Promoción del Deporte; 2018.
[22] Kim D, Ko YJ. The impact of virtual reality (VR) technology on sport spectators' flow. Computers in Human Behavior. 2018 Diciembre; 93: p. 346-356. 